\title{
Performance Evaluation of a Multi-band UWB Localization and Communication System based on Modified Gegenbauer Functions
}

\author{
A.Lamari $\dagger$, F.Elbahhar $\ddagger$, A. Rivenq $\dagger$, \\ J.M Rouvaen $\dagger$, M. Heddebaut $\ddagger$ \\ $\dagger$ IEMN-DOAE (UMR 8520 CNRS), \\ University of Valenciennes, BP311, Le Mont-Houy, \\ 59313 Valenciennes, France \\ †INRETS-LEOST, 20 rue Elisée Reclus, \\ 59650,Villeneuve-d'Ascq, France
}

\begin{abstract}
Ultra Wide Band (UWB) may offer very effecient solutions for various communication and sensing applications. Two approaches have emerged, which meet all the communication requirements: impulse and multi-band systems. The first system operates between 3.1 to $10.6 \mathrm{GHz}$ in a single frequency band. The second one exploits the division of the bandwidth into sub-bands (each sub-band width being up to around $500 \mathrm{MHz}$ wide). The present work is concerned in the second approach. So, we propose to use orthogonal functions called Modified Gegenbauer Functions (MGF) in the multi-band UWB system. Different scenarios are studied and compared in the scope of a system offering communication and location capabilities for subway trains.

In order to evaluate the performance of the proposed system, the Bit Error Rate (BER) values are calculated and analyzed in the presence of multi-user interference, assuming asynchonous users. It is shown that Gegenbauer functions offer the performance required for our multi-band UWB communication and location system.
\end{abstract}

\section{Introduction}

\subsection{UWB systems}

Ultra Wide Band communication using impulse radio (IR-UWB) has many interesting features for wireless applications and has attracted much research and industrial attention recently $[1,2]$.

UWB signals using impulse radio can be divided into two groups: single band (pulsed) systems and multi-band systems using a single carrier in each sub-band. 
The classical multi-band UWB system consists in dividing the bandwith into several sub-bands in a fixed manner or using a frequency hopping technique to address each sub-band [3]. The frequency hopping depends on pseudo-random code sequences.

Another multi-band system based on orthogonal frequency division multiplexing (OFDM) techniques on each sub-band has been proposed (MB-OFDM). During its standardisation attempt by IEEE 802.15.3a group [4], its good properties for capturing efficiently multi-path energy, mitigating interference and achieving large throughput $[5,6]$ have been demonstrated. In the MB-OFDM scheme, the signals consist of 128 sub-carriers spaced $4.125 \mathrm{MHz}$ apart and utilize the 3.1 to $10.6 \mathrm{GHz}$ range in 14 sub-bands, the bandwidth of each sub-band being set to $528 \mathrm{MHz}$. But the drawback of this type of system is that the transmitter is slightly more complex because it requires an inverse FFT (IFFT) and the peakto-average ratio may be slightly higher than that of the other pulsed multi-band approach.

All multi-band systems are attractive radio techniques that coexist with other narrowband signals and have interesting properties. For example, the information can be processed over a much smaller bandwidth, thus reducing the complexity of the design, reducing the power consumption, lowering the cost, and improving spectral flexibility and worldwide compliance. However, the disadvantage of a pulsed multi-band system is the difficulty in collecting significant multi-path energy using a single RF chain. Moreover, the MB-OFDM system requires relatively large computational power because of the required fast Fourier transform (FFT) processors.

\section{$1.2 \quad$ Studied system}

The proposed system is designed for application in subway transportation area, for simultaneous location of trains and inter-train communication.

The subway line is divided in parts called districts of about $1 \mathrm{~km}$ length. When a train is in a district, it is declared to be engaged and no coach can go in until the train leaves it. This is the safety system adopted in most of the current networks. In this scope, only a few trains (say from 2 to 4 , due to limited emitted power) will be allowed to receive or transmit data or video in any given area, including messages broadcast to passengers or security informations sent to or from control center, like train status or problems encountered on the way or on-board.

For this communication application, a sufficiently low BER, say between $10^{-4}$ and $10^{-3}$, is required. Moreover the distance between a train and a preceding one must be known to a precision in the meter range over distances higher than one hundred of meters in the train location application. The location application will not be studied in depth in this paper, only its main characteristics being taken into account in the design of the inter-train communication application. Although multi-band OFDM offers communication at data rates greatly exceeding those needed in our application, this is obtained at the detriment of range of communication (a few meters in this case, against at least one hundred of 
meters needed in our application). Multi-band OFDM will then clearly not be the solution retained in our system. But the principle of multi-band modulation has been retained, with only a few sub-bands.

In this paper, we propose to use original mathematical tools called Modified Gegenbauer Functions (MGF), derived from orthogonal polynomials [7], to achieve the multiple access needed in our application. These functions permit to replace the pseudo-random code used in code division multiple access (CDMA) and offer good performance in Bit Error Rate (BER) terms [8]. The orthogonal functions are introduced as basis functions for the pulse shapes in a multi-band system. It is shown in [9] that MGF offer better performance for multi-user UWB communication.

The orthogonality of these MGF is then exploited here to construct a multi-band UWB location and communication system and to reduce the Multi-User Interference (MUI) in asynchronous communications. The performance required for locating trains on the way calls for a signalling pulse width around 20ns, enabling in turns communication at a data rate reaching $25 \mathrm{Mbits} / \mathrm{s}$ (assuming a pulse repetition factor of $1 / 2$ ), which will be sufficient for the considered communication application. Moreover, owing to the physical difficulties of insuring a synchronous reception of all users (distant trains), an asynchronous system will be prefered here.

The paper is organized as follows. Section 2 presents the properties of MGFs. Section 3 describes the proposed UWB system using orthogonal MGFs and presents the computation of the theoretical mean BER values for the proposed system. Section 4 refines the desired characteristics for the proposed system. On this basis, MUI effects and their sources (interference from nearby subband and in-band, or co-channel, interference) are discussed in section 5. The performance of the system under maximum (4 users) and reduced (3 users) loads is then presented in section6. Finally, section 7 summarizes the results and presents future work.

\section{Modified Gegenbauer Functions}

In this section the Modified Gegenbauer Functions are presented. The use of Gegenbauer polynomials in ultra wideband communication has recently been proposed $[9,10]$ in the impulse radio (baseband)case.

The polynomials are defined on the interval $[-1,1]$ and verify the orthogonality relation:

$$
\int_{-1}^{+1} G_{m}(x) G_{n}(x) w(x) d x=0 \text { if } m \neq n,
$$

where $G_{m}$ and $G_{n}$ are Gegenbauer polynomials (of orders $m$ and $n$ ) and $w(x)$ is the weight function:

$$
w(x)=\left(1-x^{2}\right)^{\beta-1 / 2},
$$


$\beta>-1 / 2$ being a shape parameter.

The polynomials are recursively defined as:

$$
\begin{aligned}
n G_{n}(\beta, x) & =2(n+\beta-1) x G_{n-1}(\beta, x)-(n+2 \beta-2) G_{n-2}(\beta, x), \\
G_{0}(\beta, x) & =1 \\
G_{1}(\beta, x) & =2 \beta x
\end{aligned}
$$

where $n$ is the order of the Gegenbauer polynomials.

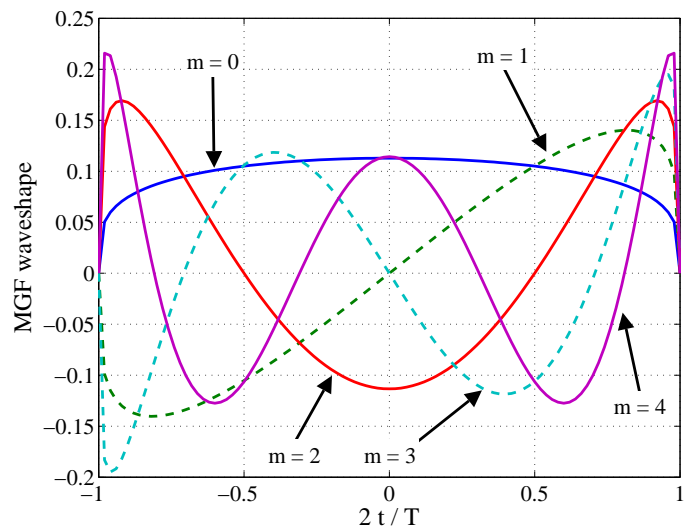

Figure 1: Time domain representation of the MGF pulses (orders 0 to 4 and $\beta=1)$.

Now, these functions are modified to become directly orthogonal in order to use them in an UWB communication system. So, the polynomials are multiplied by the square root of the weight function $w(x)$. The proposed MGF (normalized to unity energy) are thus given by:

$$
\mathcal{G}_{n}(t)=\frac{G_{n}(\beta, x) \sqrt{w(x)}}{\int_{-1}^{+1} w(u) G_{n}^{2}(\beta, u) d u} .
$$

where $x=2 t / T, T$ being the duration of all MGF waveforms.

This restricts the parameter $\beta$ to values higher than $1 / 2$ (so that the MGF waveforms $\mathcal{G}_{n}$ remain continuous at the definition interval boundaries). The corresponding functions $\mathcal{G}_{0}(t)$ to $\mathcal{G}_{4}(t)$ are drawn in Fig. 1 for $\beta=1$.

The autocorrelations of MGF waveforms have a shape much like a sinc function, peaking at a maximum value of unity (by the way of power normalization) at zero delay, see Fig. 2, and the autocorrelation main lobe width decreases slowly when $\beta$ increases. 


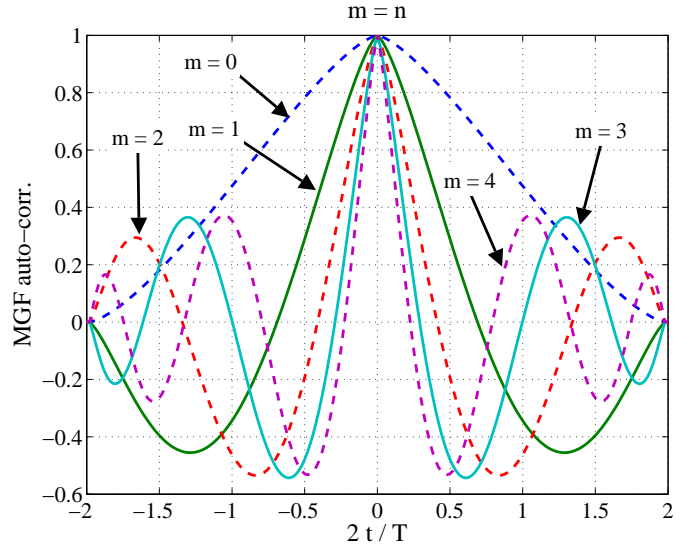

Figure 2: Time domain representation of the autocorrelation of MGF pulses $(\beta=1)$.

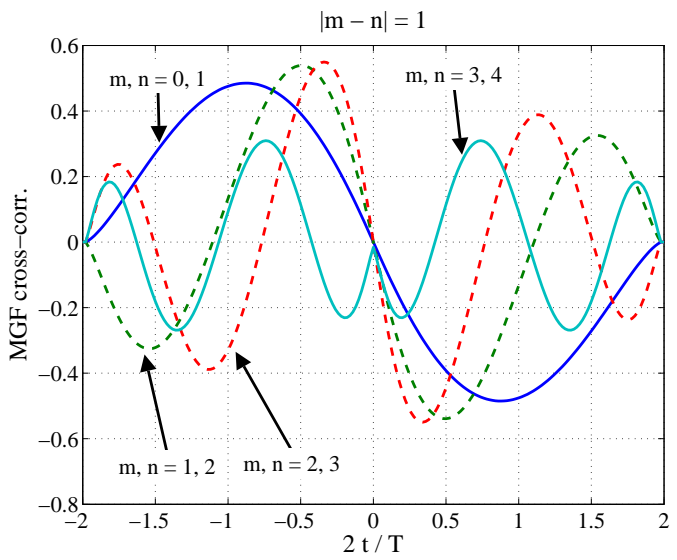

Figure 3: Time domain representation of the cross-correlation of MGF pulses consecutive orders $(\beta=1)$. 


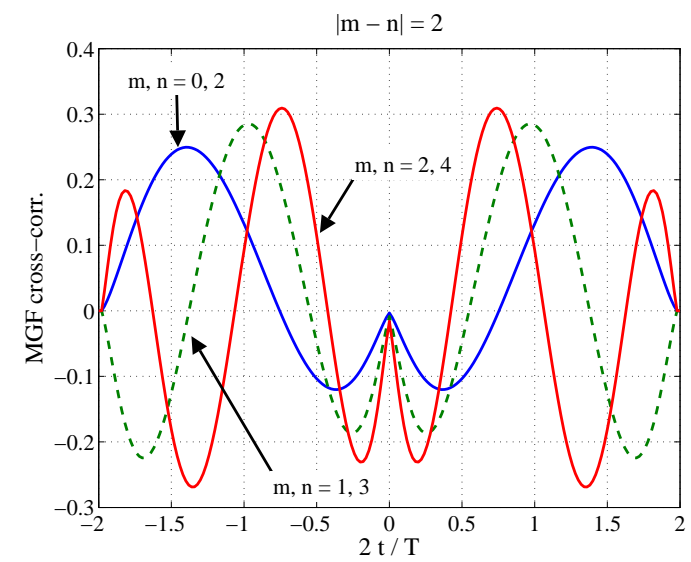

Figure 4: Time domain representation of the cross-correlation of MGF pulses non consecutive orders $(\beta=1)$.

The cross-correlations between the MGF waveforms appear to be even (resp. odd) when the pulses orders have different (resp. the same) parities and their maximum magnitudes become smaller as the difference between their orders increases, see Figs. 3 and 4.

\section{The proposed system}

\subsection{Model}

The proposed system is illustrated in Fig. 5. One specific order is attributed to each user (but the same order may eventually be given to several users occupying different sub-bands). The data for each user modulates some carrier using Binary-Phase Shift Keying (BPSK). If $\mathcal{C}_{n}(t)$ stands for the MGF attributed to the $n^{\text {th }}$ user, its transmitted signal $s_{n}$ during $k^{\text {th }}$ symbol period is expressed in baseband as:

$$
s_{n}\left(t+k T_{s}\right)= \begin{cases}\alpha_{k} \sqrt{E} \mathcal{C}_{n}(t), & |t| \leq T / 2, \\ 0 & T / 2 \leq|t|<T_{s} / 2,\end{cases}
$$

where $\alpha_{k}=\{-1,+1\}$ is the symbol sent by the user, $E$ stands for the energy of all MGF (which was normalized to unity in section 2 above), $T$ is the MGF duration and $T_{s}$ is the symbol period (assumed larger than $T$ ).

The information will be transmitted in different frequency sub-bands, using one of several possible strategies: each sub-band may be attributed exclusively to a single fixed user or eventually shared among several fixed users; alternatively, its affectation to users may be controlled via a Frequency Hopping $(\mathrm{FH})$ technique. 


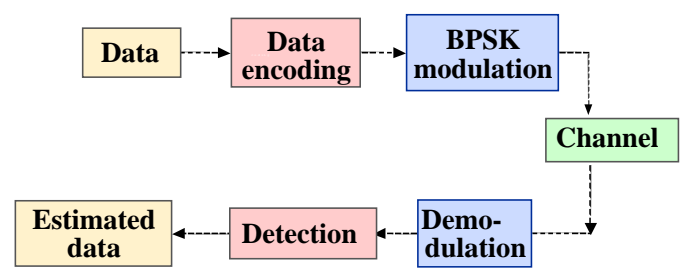

Figure 5: Block diagram of the proposed system.

In this paper, a classical simple channel model is considered as reference: the additive white Gaussian noise (AWGN) with zero mean and two sided spectral density equal to $\mathrm{N}_{0} / 2$. This is justified by the fact that no medium range UWB channel model is broadly accepted and that highly variable environments like tunnels are encountered in a railway application. The possible effects of a real channel are investigated as an extension of the present study.

Then, the signal to noise ratio (SNR) will be given by:

$$
\mathrm{SNR}=\frac{E}{N_{0}}
$$

In this study, we will assume perfect time synchronisation between the emitter and receiver for the desired user (say $i^{\text {th }}$ one). The receiver consists in a numerical correlator and the detection unit. The correlator outputs the crosscorrelation function between the received signal $r_{i}(t)$ and the template signal for the considered user $\mathcal{C}_{i}(t)$. This output is sampled at time $t=T / 2$ (if the starting time for correlation computation is taken as $t=-T / 2$ ), to get the decision variable $d_{i}$ :

$$
d_{i}=\left[\int_{-T / 2}^{t} r_{i}(t) \mathcal{C}_{i}(t) d t\right]_{t=T / 2} .
$$

Data is demodulated according to the sign of the decision variable. A data bit 1 is, for example, assumed if the sign is positive, and a data bit 0 if the sign is negative. Such a receiver constitutes an implementation of the optimal single user matched filter receiver $[15,16]$.

For the asynchronous communication with several users occupying the same sub-band, the MUI can be reduced if the cross-correlation between the signal sent by the considered user and signals sent by the other users is small. On another hand, the inter-symbol interference (ISI) can be reduced by choosing signalling waveforms with suitable autocorrelation functions (with a sufficiently high main lobe to side-lobe amplitude ratio). Such criterions are met using MGF (at least for the five lowest orders, 0 to 4 ). 
Each user sends a sub-band waveform whose carrier frequency is fixed or decided by a unique FH sequence. Due to the orthogonality of the MGF, it is possible to transmit multiple pulses with different orders simultaneously in each subband without any MUI, if the users are synchronous, and limited MUI in the asynchronous case.

\subsection{BER computation}

Let us denote the number of users by $N_{u}$. If $b_{n}= \pm 1$ stands for the symbol sent by user $n$ in a given symbol period, the signal at input of receiver for $i^{\text {th }}$ user may be written as:

$$
r_{i}=\sqrt{E}\left[b_{i} \mathcal{C}_{i}(t)+\mathrm{MUI}\right]+n(t),
$$

where $n(t)$ is an AWGN term and MUI stands for a multi-user interference term:

$$
\mathrm{MUI}=\sum_{\substack{j=1 \\ j \neq i}}^{N_{u}} b_{j} \mathcal{C}_{j}(t),
$$

where $b_{j}= \pm 1$ and $\mathcal{C}_{j}$ stand for, respectively, the symbol emitted by user $j$ in the considered symbol period and the MGF waveform atributed to user $j$.

It has been assumed that two successive symbols sent by one user never overlap with a symbol emitted by any other user. This leads, for the AWGN channel, to the condition $T_{s} \leq 2 T$, where $T_{s}$ stands for the MGF repetition period, that is the symbol period.

The decision variable for user $i$ may then be expressed as:

$$
d_{i}=E\left[b_{i}+\sum_{\substack{j=1 \\ j \neq i}}^{N_{u}} b_{j} R_{i j}\right]+N=\mathcal{M}+N .
$$

$R_{i j}$ stands for the cross-correlation between users $i$ and $j . \quad N$ is the crosscorrelation of $n(t)$ with the template signal $\mathcal{C}_{i}(t)$, that is a zero mean gaussian variable, with variance $E N_{0} / 2$. The decision variable $d_{i}$ appears then as a random gaussian variable centered around the value $\mathcal{M}$, with a variance equal to $E N_{0} / 2[15]$.

As an asynchronous system is considered, there exists an unavoidable random time delay $\tau_{i j}$ between users $i$ and $j$ (which would become a constant in a synchronous sytem, hopefully identically 0 in the perfectly synchronous case or a small constant value if synchronization is not perfect).

Moreover, the MGF waveforms are used to modulate oscillators which delivers the carriers at sub-bands centre frequencies in the multi-band system. It is not possible to maintain the phase relationship between these oscillators and a random phase difference term $\phi_{i j}$ appears between user $i$ and $j$, which would nullify in the baseband IR-UWB case. 
Finally, users $i$ and $j$ may occupy different frequency sub-bands, so that the frequency gap between them is denoted by $\Delta f_{i j}=f_{j}-f i$ (which nullifies if the two users occupy the same sub-band).

The most general expression for the cross-correlation term $R_{i j}$ is therefore:

$$
R_{i j}\left(\tau_{i j}, \phi_{i j}, \Delta f_{i j}\right)=\int_{-T / 2}^{+T / 2} \mathcal{C}_{i}(t) \mathcal{C}_{j}\left(t-\tau_{i j}\right) \cos \left[2 \pi \Delta f_{i j}\left(t-\tau_{i j}\right)+\phi_{i j}\right] d t .
$$

Following the standard procedure $[15,16]$, the BER for a given situation $\left(b_{i}, b_{j}\right.$, $\mathcal{C}_{i}, \mathcal{C}_{j}$ and $\Delta f_{i j}$ fixed, particular values of $\tau_{i j}$ and $\phi_{i j}$ ) amounts to:

$$
\operatorname{ber}_{i}=Q\left(\sqrt{2 E\left[b_{i}+\sum_{\substack{j=1 \\ j \neq i}}^{N_{u}} b_{j} R_{i j}\right]} / N_{0}\right)
$$

where the function $Q$ is related to the complementary error function and defined as:

$$
Q(x)=\frac{1}{\sqrt{2 \pi}} \int_{x}^{+\infty} \exp \left(-u^{2} / 2\right) d u .
$$

Now, let us assume that $\phi_{i j}$ is a random phase term, uniformly distributed in the interval $[-\pi,+\pi]$, which represents the unavoidable phase difference between the (independent) oscillators in the two transmitters, and that the random delay $\tau_{i j}$ between users is uniformly distributed in the $[-T,+T]$ range.

The mean BER for the considered situation $\left(b_{i}, b_{j}, \mathcal{C}_{i}, \mathcal{C}_{j}\right.$ and $\Delta f_{i j}$ fixed) may then be obtained by taking the expectation of equation (14) over all $N_{u}-1$ phases $\phi_{i j}$ and delays $\tau_{i j}(j \neq i)$. Denoting the expectation operator by $\mathcal{E}\{$.$\} ,$ this may be written as [12-14]:

$$
\overline{\operatorname{ber}}_{i}=\mathcal{E}\left\{\operatorname{ber}_{i}\right\}_{\tau_{i j}, \phi_{i j}}, \text { for } j=1,2, \ldots, N_{u}, j \neq i .
$$

In principle, there remains to repeat the procedure for all bits possibly emitted by all users and take the average of the corresponding results (assuming that the bits 0 and 1 are equiprobably sent by each user) to get the mean BER for user $i$ in a given scenario $s_{k}\left(\mathcal{C}_{i}, \mathcal{C}_{j}\right.$ and $\Delta f_{i j}$ fixed $)$, denoted by $\overline{\operatorname{ber}}_{i, s_{k}}$.

The last step will be to take the mean over all scenarios which may be encountered (by varying in some manner the MGFs and sub-bands attributed to the users) for a given number of users $N_{u}$, which will be denoted as $\mathrm{BER}_{i}$.

$$
\operatorname{BER}_{i}=\frac{1}{N_{\text {scen }}} \sum_{k=1}^{N_{\text {scen }}} \overline{\operatorname{ber}}_{i, s_{k}},
$$

where $N_{\text {scen }}$ stands for the number of possible scenarios. 
All above BER values may also be compared to that obtained for an optimal single user receiver [15]:

$$
\mathrm{BER}_{\mathrm{opt}}=Q\left(\sqrt{\frac{2 E}{N_{0}}}\right),
$$

\subsection{Properties of the $R_{i j}$ cross-correlation terms}

From the presence of the cosinus term in the cross-correlation expression of equation (13) and the randomness of the term $\phi_{i j}$ in its argument, one may deduce some properties that lessen the computations involved in the BER estimation procedure from equation (17).

One may first notice that, to each value of the cosinus term (corresponding to some value of $\phi_{i j}$ ), one may associate its opposite (corresponding to $\phi_{i j} \pm \pi$ ). Then, the contribution of the bit sent by each user $j$ to the final BER will be the same (whenever the corresponding symbol be +1 or -1 ). Then the step of taking an average over all possible bit configurations may be avoided and all sent bits may be arbitrarily set to $1\left(b_{n}=1\right.$, for $\left.n=1,2, \ldots, N_{u}\right)$.

Now, by taking the expectation over $\phi_{i j}$ first (before that on $\tau_{i j}$ ), one sees that the additive term $-2 \pi \Delta f_{i j} \tau_{i j}$ may be incorporated into the random phase $\phi_{i j}$ (i.e. it has no effect on the final result). The only effect of $\tau_{i j}$ results then from the delayed MGF code $\mathcal{C}_{j}$. Moreover, if users $i$ and $j$ share the same sub-band, the cosinus term reduces to $\cos \left(\phi_{i j}\right)$, which may be taken out from the integral in the expression of $R_{i j}$.

One may also see that $R_{i j}\left(\tau_{i j}, \phi_{i j}, \Delta f_{i j}\right)=R_{i j}\left(\tau_{i j},-\phi_{i j},-\Delta f_{i j}\right)$ from equation (13), so that only the absolute value of the frequency gap plays a role in the final BER results.

The expression of $R_{i j}$ in equation (14) may now be replaced by:

$$
R_{i j}\left(\tau_{i j}, \phi_{i j}, \Delta f_{i j}\right)=\int_{-T / 2}^{+T / 2} \mathcal{C}_{i}(t) \mathcal{C}_{j}\left(t-\tau_{i j}\right) \cos \left(2 \pi\left|\Delta f_{i j}\right| t+\phi_{i j}\right) d t .
$$

Finally, although not apparent at first blush in equation (13), it may be shown that there is symmetry between users $i$ and $j$, that is the contribution of user $i$ to final BER for user $j$ is identical to that of user $j$ to final BER for user $i$ (mainly using changes in variables and noting that $\tau_{i j}=-\tau_{j i}, \phi_{i j}=-\phi_{j i}$ and $\Delta f_{i j}=-\Delta f_{j i}$. This will simplify the computation of BER when dealing with several users. 


\section{Location and communication system charac- teristics}

The parameters of the proposed simultaneous location and communication system will now be precised.

\subsection{Communication}

The frequency range from 3 to $6 \mathrm{GHz}$ will be used for our system. In order to support a maximum of 4 users and to cope with smaller numbers of users, 2 sub-bands may be used for communication, with 0,1 or 2 user(s) sharing each given sub-band. This implies the use of two different MGF codes in the system. One may choose, for example, to put these two communication sub-bands in the spectrum region higher than $4 \mathrm{GHz}$.

To get a maximum data flow rate of $25 \mathrm{Mbits} / \mathrm{s}$, one MGF must be emitted each 40ns, so that the MGF duration $T$ (taken equal to half the symbol period $T_{s}$ ) will be around $20 \mathrm{~ns}$. Each user may be represented by a doublet $(m, p)$, where $m$ is the order of the MGF attributed to him and $p=1$ or 2 , in increasing frequency order, represents the sub-band it uses.

The most logical MGF order and sub-band affectation strategy, taking maximum system load (4 users) into account is as follows:

- one MGF code (say $\mathcal{G}_{m}$ ) is reserved to trains (from 0 to 2 ) going in one direction, the other code $\left(\mathcal{G}_{n}\right)$ being attributed to trains (also from 0 to 2) going in the opposite one,

- the two sub-bands are alternatively assigned to consecutive trains on the same side.

Several variants of the system may then be defined and studied: two different MGF codes are to be chosen among $\mathcal{G}_{0}$ to $\mathcal{G}_{4}$ (for which the generators are considered as realizable) and the frequency separation between the to sub-bands $\Delta f$ may be varied. For this latter purpose, a dimensionless parameter $M=$ $\Delta f \times T$ may be introduced.

In fact, it may be expected that BER from equ. (17) is always greater than or equal to $\mathrm{BER}_{\text {opt }}$ from equ. (18). The equality may hold for several values of $M$ and given $(m, n)$ pairs if $R_{i j}$ from equ. (13) nullifies or remains much smaller than unity for all values of $\phi_{i j}$. This corresponds clearly to a minimal BER situation and a waveform orthogonality condition for the two nearby subbands.

\subsection{Location}

The location function may be performed using some kind of time multiplexing between the two intended system functions. This solution will call for the introduction of a caller-sender handshake protocol and reduce noticeably the communication flow rate. 
Alternatively, the location function may use two other separate sub-bands, in the low range of UWB spectrum, say around $3.5 \mathrm{GHz}$. The high frequency separation will then help to diminish interferences between the two system functions.

For location, a transponding operation mode will be used in both cases: different frequency sub-bands are reserved for callers (trains which wish to locate their positions) and the receivers-senders (the answering base stations, synchronized using satellite timing signals). The system will work on the basis of different pseudo-random codes (one by client train), each chip being materialized by a MGF. The influence of parasitic responses (from reflections on obstacles or emissions from trains not concerned in the localization process) will then be significantly diminished. The time difference of arrival (TDOA) willl be used to compute the train positions.

The simplest implementation (which avoids the deployment of a number of beacons for triangulation) is that based on the round trip time measurement: from the time elapsed between the call and the answer, one may deduce (taking the eventual processing, coding and decoding times in electronics into account) the distance between caller and answerer, if the electromagnetic wave celerity is known.

\section{$5 \quad$ Study of the MUI effects}

Several problems have to be considered. Firstly, the interferences between users sharing the same sub-band must be studied. Second, one must also address the problem of interferences between users in nearby sub-bands.

\subsection{Several users in the same sub-band}

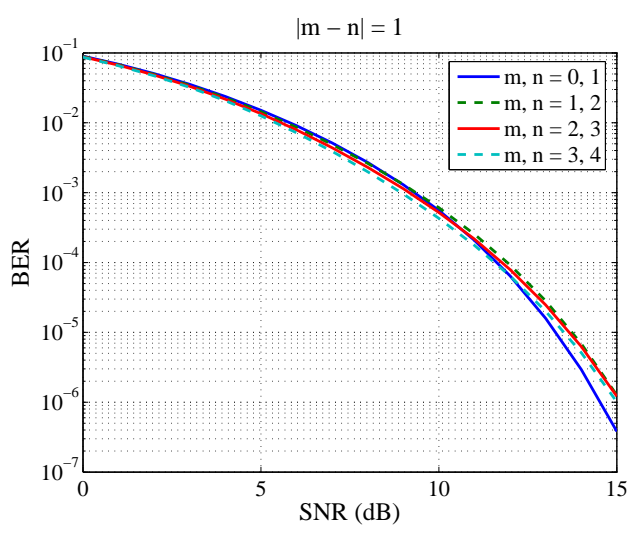

Figure 6: Two users per sub-band $-|m-n|=1$.

The problem is bound to the possible co-existence of several MGFs, all with 
Table 1: BER for two users in same sub-band $-\mathrm{SNR}=10 \mathrm{~dB}$.

\begin{tabular}{|cccccc|}
\hline$m$ & $n=0$ & $n=1$ & $n=2$ & $n=3$ & $n=4$ \\
\hline 0 & $1.743510^{-2}$ & $5.577510^{-4}$ & $2.285310^{-5}$ & $7.720210^{-6}$ & $5.844410^{-6}$ \\
1 & $5.577510^{-4}$ & $7.006310^{-3}$ & $6.088810^{-4}$ & $3.379610^{-5}$ & $1.289610^{-5}$ \\
2 & $2.285310^{-5}$ & $6.088810^{-4}$ & $4.414710^{-3}$ & $5.199410^{-4}$ & $4.377810^{-5}$ \\
3 & $7.720210^{-6}$ & $3.379610^{-5}$ & $5.199410^{-4}$ & $3.139510^{-3}$ & $4.290110^{-4}$ \\
4 & $5.844410^{-6}$ & $1.289610^{-5}$ & $4.377810^{-5}$ & $4.290110^{-4}$ & $2.374010^{-3}$ \\
\hline
\end{tabular}

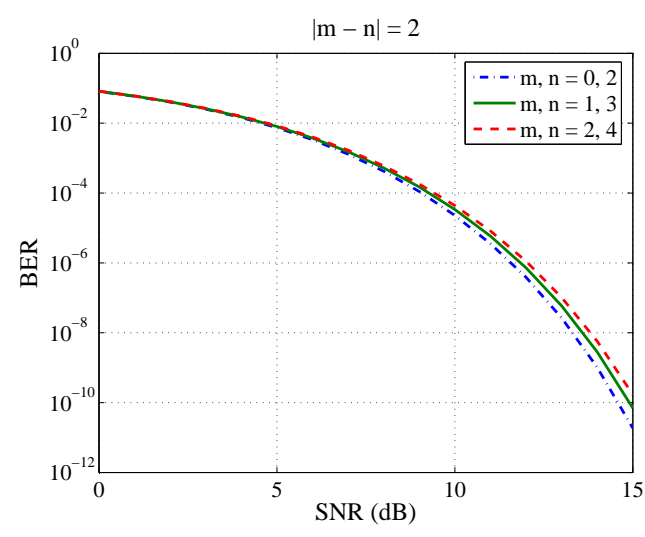

Figure 7: Two users per sub-band $-|m-n|=2$.

different orders in the same sub-band. The same sub-band is then shared by several users, as theoretically enabled by MGF orthogonality. Let us consider the case of two MGFs, with orders $m$ and $n$, sharing the same sub-band.

The cross-correlation term in equation (19) reduces to:

$$
R_{i j}\left(\tau_{i j}, \phi_{i j}, \Delta f_{i j}\right)=\cos \left(\phi_{i j}\right) \int_{-T / 2}^{+T / 2} \mathcal{G}_{m}(t) \mathcal{G}_{n}\left(t-\tau_{i j}\right) d t .
$$

The BER has been computed from equation (17), using the cross-correlation expression (20) above, for different SNR and $\beta$ values. As may be expected, the results obtained for two identical MGF orders $(m=n)$ are unsatisfactory: the BER remains always greater than $10^{-3}$, even at higher SNR values.

Fig. 6 and Fig. 7 correspond to the cases $|m-n|=1$ and $|m-n|=2$, respectively. One clearly sees that the latter case is more interesting in BER terms as the former one.

Satisfactory solutions for low (lower than $10^{-4}$ ) BER communication are found for each $(m, n)$ pair with $m \neq n$, as illustrated in table 1 . Moreover, the BER is a decreasing function of the difference betweens the two orders $|m-n|$.

There will be at most two users with the same MGF waveform in each sub-band 


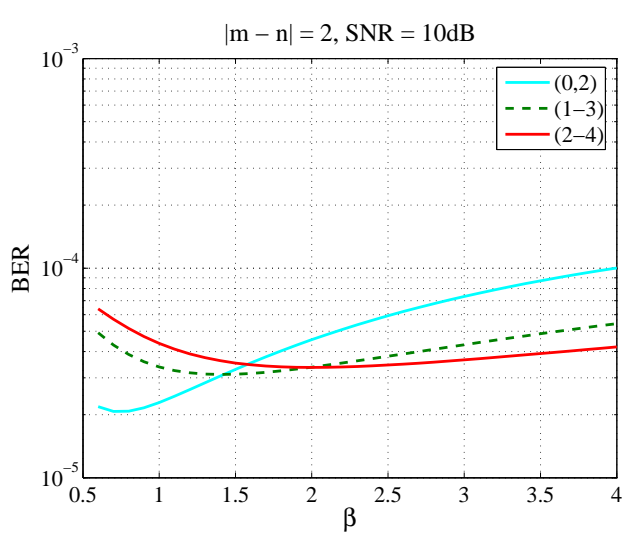

Figure 8: Two users per sub-band - Influence of parameter $\beta$.

in our proposed system. In this case, all $(m, n)$ pairs are possible candidates for their orders, but the BER decreases more and more as the difference in these orders, $|m-n|$, increases.

Thus, the solution $|m-n|=2$ has been prefered for our system: it gives better performance than the $|m-n|=1$ solution and more design flexibility (3 alternatives)than the more performing $|m-n|=3$ (2 alternatives) and $|m-n|=4$ (a single alternative) solutions. Therefore only the alternative orders pairs $(m, n)=(0,2)$ or $(1,3)$ or $(2,4)$ will be considered in the following. The results above were obtained by fixing the value of the parameter $\beta$ to unity. This parameter has also been varied to study its influence on the BER. As illustrated in figure 8 , the most noticeable finding is the existence of a minimum in BER for some $\beta$ value between 0.7 and 2, depending on the MGF code pair considered. However all BER values are not too severely modified when $\beta$ varies and the order pairs $|m-n|=2$ remain superior (from the BER performance viewpoint) to $m=n$ pairs.

\subsection{Interferences between nearby sub-bands}

The mutual interference of MGFs emitted in different sub-bands will be studied here. The key parameter (apart of the involved MGF orders) is the product $M=T \Delta F$ of the MGF time duration $T$ and the absolute value of the frequency spacing between the two sub-bands $\Delta F$.

Let us assume that the two nearby sub-bands are each occupied by a single user (MGF). The users are assumed to be received with equal powers and use the odrder pairs defined in section 5.1 above. The two users may use the same MGF $(m=n)$ or the pair of MGFs $(|m-n|=2)$.

The cross-correlation term in equation (19) reduces to: 


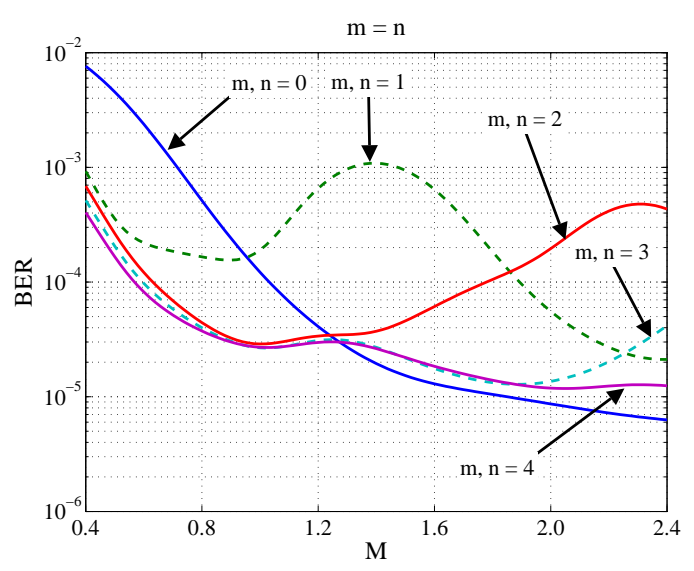

Figure 9: Single user per sub-band $-m=n$.

$$
R_{i j}\left(\tau_{i j}, \phi_{i j}, \Delta f_{i j}\right)=\int_{-T / 2}^{+T / 2} \mathcal{G}_{m}(t) \mathcal{G}_{n}\left(t-\tau_{i j}\right) \cos \left(2 \pi M t / T+\phi_{i j}\right) d t
$$

The BER has been computed from equation (17), using the cross-correlation expression (21), for different SNR values and several values of parameter $\beta$ (which controls the MGF waveshapes, as described in section 2). The results obtained for $\mathrm{SNR}=10 \mathrm{~dB}$ and $\beta=1$ are shown in the following figures, where the parameter $M$ is kept lower than 2.4 (which corresponds to $\Delta f=120 \mathrm{MHz}$ with the value chosen for $T$ ).

Figures 9 and 10 give the results obtained for, respectively, $m=n$ and $\mid m-$ $n \mid=2$. A number of configurations enabling communication at low BER are observed. The curves have an oscillatory character, but the minima are not equal nor very close to $\mathrm{BER}_{\mathrm{opt}}$.

It may also be noticed that, when increasing the value of parameter $M$, the BER curves for all $(m, n)$ pairs keep their oscillatory behavior, but have the same asymptotic limit equal to the BER of the optimal single user receiver $\left(\mathrm{BER}_{\mathrm{opt}}\right)$, as shown for example in figures 11 and 12 , for respectively, $m=n$ and $|m-n|=2$. It may then be concluded that satisfactory solutions for low BER communication exist finally for each $(m, n)$ pair. The ranges of $M$ values (for $0.4<M<2.4$ ) leading to a BER lower than $10^{-4}$ are summarized in table 2, for the cases $m=n$ and $|m-n|=2$ retained in previous section 5.1.

The influence of parameter $\beta$ has again been studied. As illustrated in figure 13, it is found that, for $M$ values higher than 1.4, the BER values increase with $\beta$. In fact, the main efect is a shifting of the BEr curve towards higher $M$ values. 


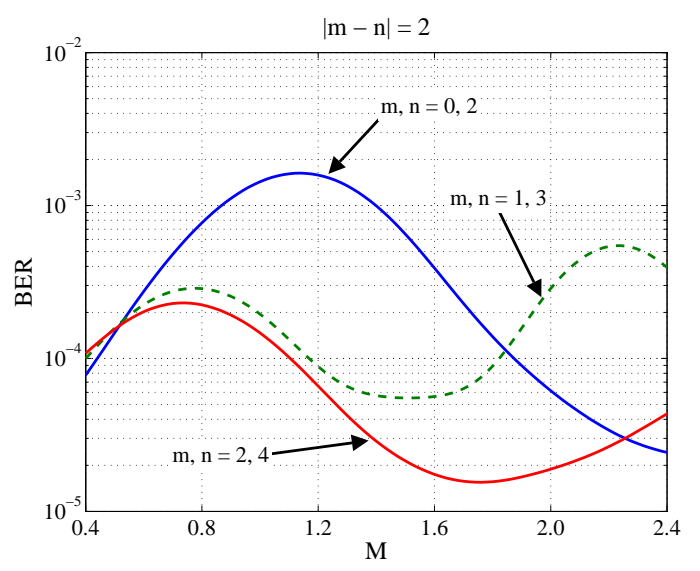

Figure 10: Single user per sub-band $-|m-n|=2$.

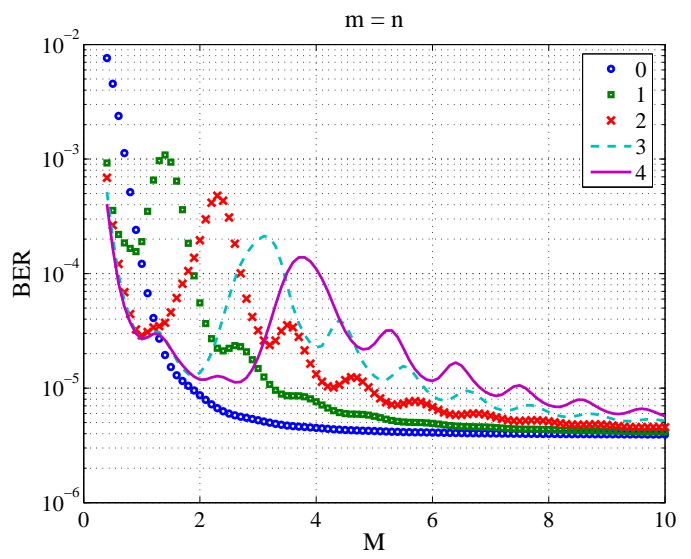

Figure 11: Asymptotic BER limit $-m=n$. 


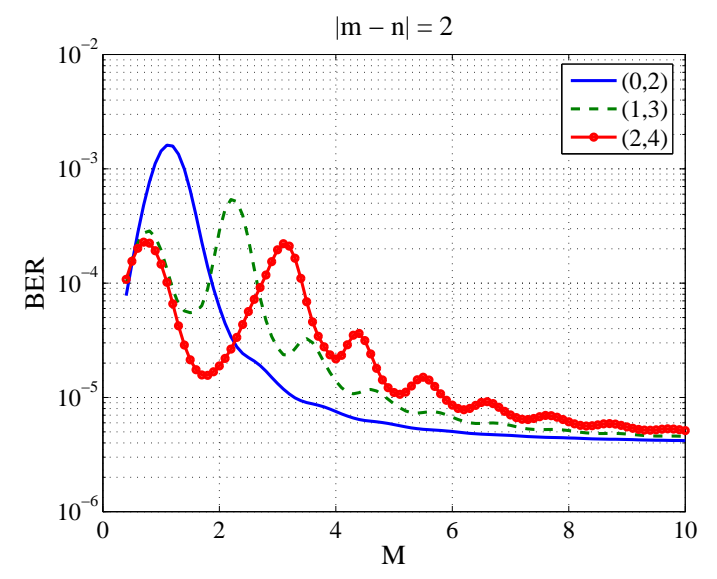

Figure 12: Asymptotic BER limit $-|m-n|=2$.

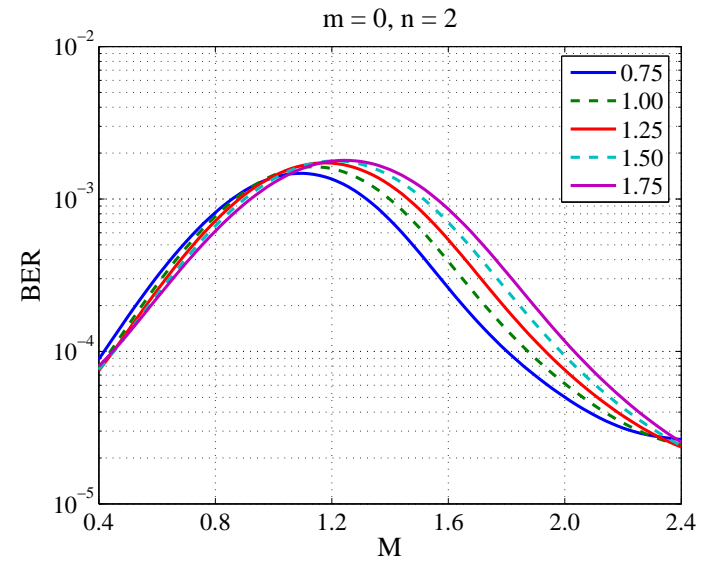

Figure 13: Two users in sub-band - Influence of parameter $\beta-(m, n)=(0,2)$. 
Table 2: Available ranges of $M$ values $-\mathrm{SNR}=10 \mathrm{~dB}$

\begin{tabular}{cc}
\hline Orders $(m, n)$ & Ranges \\
\hline$(0,0)$ & {$[1.04,2.40]$} \\
$(1,1)$ & {$[1.90,2.40]$} \\
$(2,2)$ & {$[0.64,1.76]$} \\
$(3,3)$ & {$[0.60,2.40]$} \\
$(4,4)$ & {$[0.58,2.40]$} \\
\hline$(0,2)$ & {$[1.88,2.40]$} \\
$(1,3)$ & {$[1.18,1.82]$} \\
$(2,4)$ & {$[1.12,2.40]$} \\
\hline
\end{tabular}

\subsection{Conclusion}

From the results of the above section 5.1, the orders of the 2 MGF co-existing in each sub-band are chosen of the same parity, say $(0,2),(1,3)$ or $(2,4)$. It is desired to preserve spectral efficiency: the frequency spacing between subbands (and thus parameter $M$ ) must be as low as possible (without sacrifying performance). From figures 11 and 12 of section 5.2, one sees that the frequency gap $\Delta f$ between centres of sub-bands may be tuned, for our final system, in the 80 to $200 \mathrm{MHz}$ range $(M \in[1.6,4.0])$. This implies a full frequency range for the communication function which will not exceed $400 \mathrm{MHz}$, spanning for example from $4.6 \mathrm{GHz}$ to $5.0 \mathrm{GHz}$. Moreover, the parameter $\beta$ will be fixed to unity in the following, a good trade-off between results of subsections 5.1 and 5.2 above.

\section{System performance}

The performance has been evaluated for the proposed system using two nearby sub-bands with a frequency separation $\Delta f$, with MGFs $\mathcal{G}_{m}$ and $\mathcal{G}_{n}$ used in each sub-band. The three following cases have been tested:

- system 1: $(m, n)=(0,2)$,

- system 2: $(m, n)=(1,3)$,

- system 3: $(m, n)=(2,4)$.

For each of the three defined systems, there are thus 8 different scenarios: 2 choices for $m$ (index $i_{1}=1,2$ ), 2 choices for sub-band of leading train on one way side (lower, upper, index $i_{2}=1,2$ ) and on the opposite side (lower, upper, index $\left.i_{3}=1,2\right)$. The configuration numbering may then follow the scheme $i_{S}=4\left(i_{1}-1\right)+2\left(i_{2}-1\right)+i_{1}$.

There are only 4 cross-correlations terms possibly involved in the MUI expression for each scenario: 


$$
\begin{aligned}
R_{a}(\tau, \phi) & =\cos (\phi) \int_{-T / 2}^{+T / 2} \mathcal{G}_{m}(t) \mathcal{G}_{n}(t-\tau) d t \\
R_{b}(\tau, \phi) & =\int_{-T / 2}^{+T / 2} \mathcal{G}_{m}(t) \mathcal{G}_{n}(t-\tau) \cos (2 \pi M t / T+\phi) d t \\
R_{c}(\tau, \phi)= & \int_{-T / 2}^{+T / 2} \mathcal{G}_{m}(t) \mathcal{G}_{m}(t-\tau) \cos (2 \pi M t / T+\phi) d t \\
R_{d}(\tau, \phi)= & \int_{-T / 2}^{+T / 2} \mathcal{G}_{n}(t) \mathcal{G}_{n}(t-\tau) \cos (2 \pi M t / T+\phi) d t
\end{aligned}
$$

Before considering the system at full load, the most probable, 3 users, configuration will be studied.

\subsection{Most probable configuration: 3 communicating trains}

The most probable situation has been tested first. Here three trains are involved:

- two trains (a leading one and a following one, denoted respectively as users 1 and 2) on one way side, sharing the MGF $\mathcal{G}_{u 1, u 2}\left(=\mathcal{G}_{m}\right.$ or $\left.\mathcal{G}_{n}\right)$, in two nearby sub-bands,

- the third train on the opposite side (user 3), using the MGF $\mathcal{G}_{u 3}\left(=\mathcal{G}_{n}\right.$ or $\left.\mathcal{G}_{m}\right)$, in any of the two sub-bands.

It is readily seen that the MUI terms for user 3 are all identical, so that the averaging over all scenarios is not needed in this case and the BER for user 3 may be written as:

$$
\mathrm{BER}_{3}=\mathcal{E} \cup\left\{Q\left(\sqrt{2 E\left[1+R_{a}\left(\tau_{1}, \phi_{1}\right)+R_{b}\left(\tau_{2}, \phi_{2}\right)\right]} / N_{0}\right)\right\},
$$

where the subscript notation $\cup$ means that expectation is taken over all $\tau$ and $\phi$ variables $\left(\tau_{1}, \tau_{2}, p h i_{1}, \phi_{2}\right.$ here).

By considering the equalities betweenthe MUI terms for all scenarios, it may also be shown that the BER for users 1 and 2 are identical and given by :

$$
\begin{aligned}
\mathrm{BER}_{1-2, m}= & \frac{1}{2} \mathcal{E}_{\cup}\left\{Q\left(\sqrt{2 E\left[1+R_{c}\left(\tau_{1}, \phi_{1}\right)+R_{a}\left(\tau_{2}, \phi_{2}\right)\right] / N_{0}}\right)+\right. \\
& \left.Q\left(\sqrt{2 E\left[1+R_{c}\left(\tau_{1}, \phi_{1}\right)+R_{b}\left(\tau_{2}, \phi_{2}\right)\right] / N_{0}}\right)\right\},
\end{aligned}
$$

if they use the MGF $G_{m}$, and : 


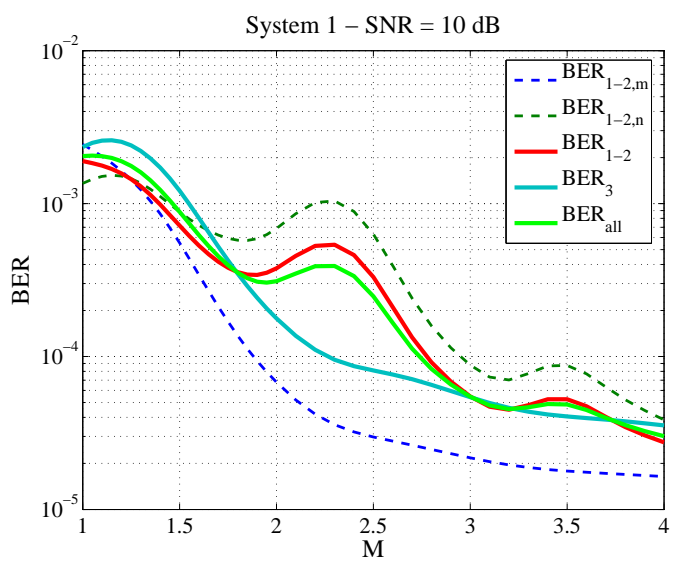

Figure 14: BER vs. $M-\mathrm{SNR}=10 \mathrm{~dB}-m, n=0,2$.

$$
\begin{aligned}
\operatorname{BER}_{1-2, n}= & \frac{1}{2} \mathcal{E}_{\cup}\left\{Q\left(\sqrt{2 E\left[1+R_{d}\left(\tau_{1}, \phi_{1}\right)+R_{a}\left(\tau_{2}, \phi_{2}\right)\right] / N_{0}}\right)+\right. \\
& \left.Q\left(\sqrt{2 E\left[1+R_{d}\left(\tau_{1}, \phi_{1}\right)+R_{b}\left(\tau_{2}, \phi_{2}\right)\right] / N_{0}}\right)\right\}
\end{aligned}
$$

if they use the MGF $G_{n}$ instead.

The mean BER for the users 1 and 2 is then:

$$
\mathrm{BER}_{1-2}=\left(\mathrm{BER}_{1,2, m}+\mathrm{BER}_{1-2, n}\right) / 2 .
$$

The mean BER (taken over all users) is:

$$
\mathrm{BER}_{\text {all }}=\left(\mathrm{BER}_{1-2, m}+\mathrm{BER}_{1-2, n}+\mathrm{BER}_{3}\right) / 3 .
$$

The BER performance of the system may be characterized, for each of the $3 \mathrm{MGF}$ code pairs, by the values $\mathrm{BER}_{1-2, m}, \mathrm{BER}_{1-2, n}, \mathrm{BER}_{1-2}, \mathrm{BER}_{3}$ and $\mathrm{BER}_{\text {all }}$.

The influence of parameter $M$ (in the 1 to 4 range) on the BER has been studied for all three versions of the system for SNR $=10 \mathrm{~dB}$ and shown in figures 14,15 and 16. In all these curves, it may first be noticed that the overall BER (BER all $)$ is always close from the mean BER for the 2 users which are on the same side of the way $\left(\mathrm{BER}_{1,2}\right)$. From figure 16 , one sees that the BER does not decrease rapidly with $M$ for system 3 , the value $M=1.6(\Delta f=80 \mathrm{MHz})$ minimizing the mean overall BER. From figures 14 and 15, one may choose values of $M$ leading to a BER close to $10^{-4}$, like $M=2.8(\Delta f=140 \mathrm{MHz})$ for system 1 and $M=3.6(\Delta f=180 \mathrm{MHz})$ for system 2 . The corresponding overall mean BER versus SNR curves are shown in figure 17 and all BER values for SNR $=10 \mathrm{~dB}$ are given in table 3 . 


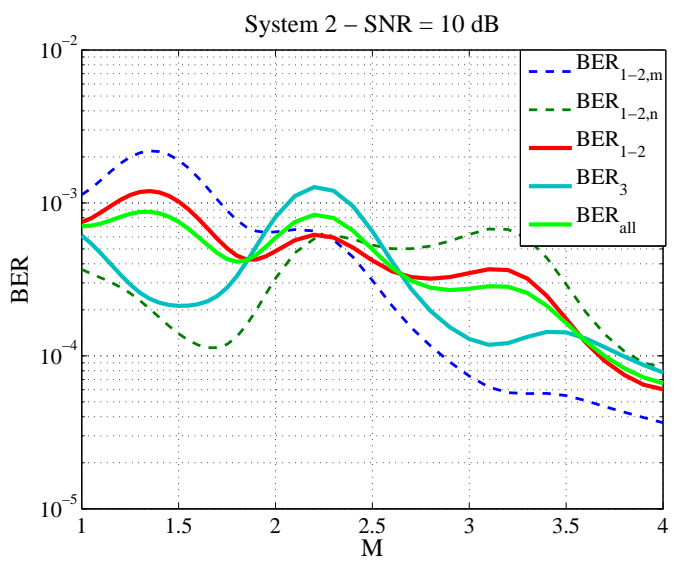

Figure 15: BER vs. $M-\mathrm{SNR}=10 \mathrm{~dB}-m, n=1,3$.

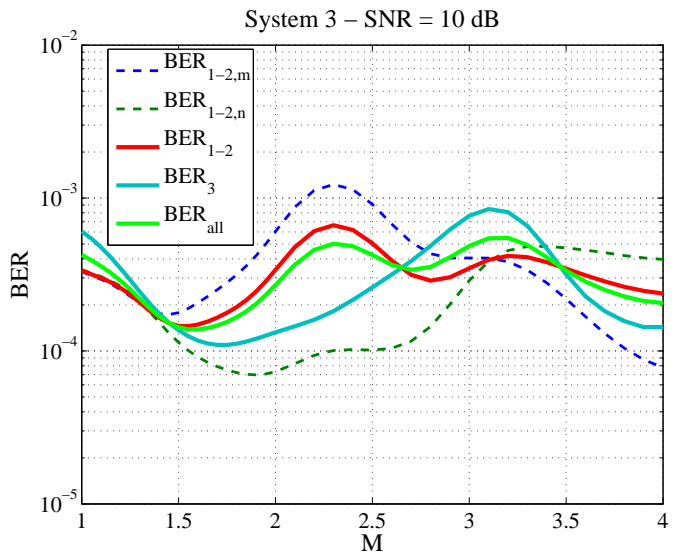

Figure 16: BER vs. $M-\mathrm{SNR}=10 \mathrm{~dB}-m, n=2,4$.

Table 3: BER for 3 users $-\mathrm{SNR}=10 \mathrm{~dB}$.

\begin{tabular}{|cccc|}
\hline BER & System 1 & System 2 & System 3 \\
\hline BER $_{1-2, m}$ & $2.470010^{-5}$ & $5.105210^{-5}$ & $2.063910^{-4}$ \\
BER $_{1-2, n}$ & $9.122610^{-5}$ & $1.944410^{-4}$ & $5.105210^{-5}$ \\
BER $_{1-2}$ & $9.170010^{-5}$ & $1.227510^{-4}$ & $1.488110^{-4}$ \\
BER $_{3}$ & $6.533310^{-5}$ & $1.300910^{-4}$ & $1.169110^{-4}$ \\
BER $_{\text {all }}$ & $8.291110^{-5}$ & $1.251910^{-4}$ & $1.381810^{-4}$ \\
\hline
\end{tabular}




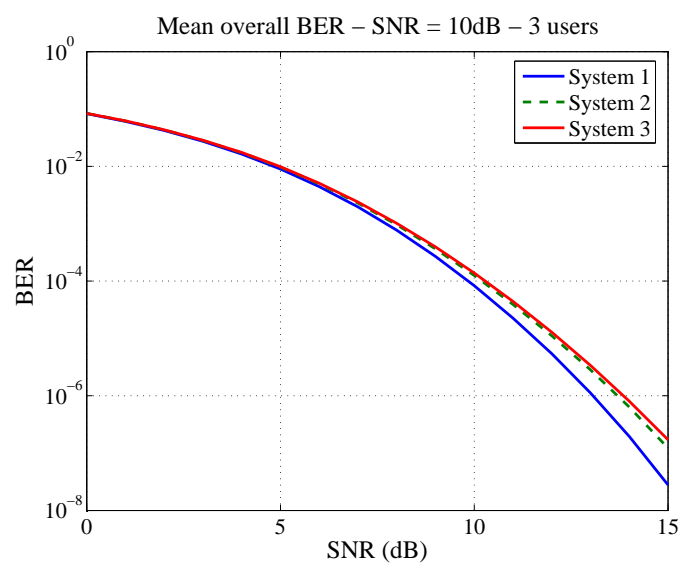

Figure 17: Mean overall BER vs. SNR - 3 users.

It may be concluded that the proposed system is fully functional for 3 users, with some degree of flexibility in the choice of its parameters (MGF code pairs, $M$ values).

\subsection{System at full load}

Let us now consider the system at full load (4 users). Following the same procedure as in subsection 6.1 above, one may show that the users which travel in the same direction have the same BER. There are then 3 figures which characterize the performance: $\mathrm{BER}_{+d}$, the BER for the 2 users sharing MGF code $\mathcal{G}_{m}$, that, $\mathrm{BER}_{-d}$, for the other 2 sharing MGF code $\mathcal{G}_{n}$ (the relation $m<n$ is assumed here), and $B E R_{\text {mean }}$ the mean BER over all 4 users. Using again the subscript notation $\cup$ to mean that expectation is taken over all $\tau$ and $\phi$ variables $\left(\tau_{1}, \tau_{2}, \tau_{3}, \phi_{1}, \phi_{2}, \phi_{3}\right.$ here), these quantities may be written as:

$$
\begin{aligned}
& \mathrm{BER}_{+d}=\mathcal{E}_{\cup}\left\{Q\left(\sqrt{2 E\left[1+R_{a b}\left(\tau_{1}, \phi_{1}, \tau_{2}, \phi_{2}\right)+R_{c}\left(\tau_{3}, \phi_{3}\right)\right] / N_{0}}\right)\right\}, \\
& \mathrm{BER}_{-d}=\mathcal{E}_{\cup}\left\{Q\left(\sqrt{2 E\left[1+R_{a b}\left(\tau_{1}, \phi_{1}, \tau_{2}, \phi_{2}\right)+R_{d}\left(\tau_{3}, \phi_{3}\right)\right] / N_{0}}\right)\right\},
\end{aligned}
$$

where:

$$
R_{a b}\left(\tau_{1}, \phi_{1}, \tau_{2}, \phi_{2}\right)=R_{a}\left(\tau_{1}, \phi_{1}\right)+R_{b}\left(\tau_{2}, \phi_{2}\right) .
$$

The mean overall BER is then $\mathrm{BER}_{\text {all }}=\left(\mathrm{BER}_{+d}+\mathrm{BER}_{-d}\right) / 2$.

The BER performance of the system at full load (4 users) has been studied for the 3 systems, adopting the same value of parameter $M$ used in subsection 6.1 above for each system. 
Table 4: BER for 4 users $-\mathrm{SNR}=10 \mathrm{~dB}$.

\begin{tabular}{|cccc|}
\hline BER & System 1 & System 2 & System 3 \\
\hline BER $_{-d}$ & $1.115910^{-4}$ & $3.719910^{-4}$ & $5.990110^{-4}$ \\
BER $_{+d}$ & $4.650010^{-4}$ & $8.825410^{-4}$ & $2.473110^{-4}$ \\
BER $_{\text {all }}$ & $2.882910^{-4}$ & $6.272710^{-4}$ & $4.231610^{-4}$ \\
\hline
\end{tabular}

The results for $\mathrm{SNR}=10 \mathrm{~dB}$ are summarized in table 4 .

These results may be compared to those obtained in the situation where all users share the same sub-band (with a carrier, i.e. not baseband), but have different MGF codes. In such case, it may be expected that all users have different BERs. Let us assume that the MGF $\mathcal{G}_{i-1}$ is attributed to user $i(i=1,2,3,4)$. There are then 6 different cross-correlations into the MUI terms of the 4 users, which take (assuming $i \neq j$ ) the form:

$$
R_{i j}(\tau, \phi)=\cos (\phi) \int_{-T / 2}^{+T / 2} \mathcal{G}_{i-1}(t) \mathcal{G}_{j-1}(t-\tau) d t,
$$

from which the BER of each user may be computed, following the method used in previous sections.

If $\mathrm{BER}_{i}$ denotes the BER for user $i$ and $\mathrm{SNR}=10 \mathrm{~dB}$, then $\mathrm{BER}_{1}=1.286410^{-3}$, $\mathrm{BER}_{2}=6.795410^{-3}$ and $\mathrm{BER}_{3}=\mathrm{BER}_{4}=1.200310^{-2}$. This clearly shows the superiority of our multi-band design, which clearly match the specifications for the communication application.

\section{Conclusion}

In this paper, a multi-band UWB multiple access system, using MGF pulses in an AWGN channel, has been proposed. The presented results show that the MGF-UWB asynchronous system gives good performance in terms of BER, with reduced MUI, particularly in the most common case of 3 communicating trains. The proposed system has many potentials advantages in terms of complexity and implementation.

It remains to study the location application in more depth and, particularly, its interactions with the communication one. The BER constraints for location are less stringent than for communication since several consecutive pulses may be used in the process, which is more apparented to a detection problem. So the probabilities of true detection and false alarm would be more significant in this case. Moreover, it has been said that the location application will use other sub-bands, lying in a widely separated frequency part of the spectrum. The problem is thus to choose, for location, a frequency separation and MGF codes, so that the impact on the BER for communication will not be significant.

It may be noticed that several phenomena have not been taken into account and must be addressed in future work. 
Firstly, the AWGN channel is only a crude approximation of a real UWB channel. In a transportation application, the channel is highly variable: the situations are very different in tunnel and outdoor parts. The theoretical results presented in this article represent then only an optimum, which may be only approached by resorting to (blind or supervised) channel equalization techniques $[17,18]$, to be developed. In parallel typical channel models (theoretical or emanating from experiments) may be established.

The matched filter receiver is not optimal in the scope of multi-user communications. More involved systems (multi-user receivers) may be developped to lower the BER, like in CDMA communications or, alternatively, error control codes may be developed [19-21].

Another key point is the Doppler effect which may be encountered in practice in our multi-band (that is multi-carrier) system. This call for the development of frequency and phase correction techniques dedicated to UWB.

Moreover, it has been assumed that all users were received with the same power. This implies the definition of a power control technique [22] in the physical (PHY) and medium access control (MAC) layers. Alternatively, near-far effects have been ignored: a close to receiver, higher power, user may forbid the detection of a far, lower power, user [23,24]. Power control and near-far effects have been the subject of a number of works in the field of CDMA communications and some of the solutions given may be adapted to UWB.

\section{References}

[1] Benedetto, M.-G., and B.R. Vojcic, "Ultra Wide Band (UWB) Wireless Communications: A Tutorial," Journal of Communication and Networks, Special Issue on Ultra-Wideband Communications, Volume: 5, Issue: 4 (December 2003), 290-302

[2] R. A. Scholtz, "Mutiple Access with Time Hopping Impulse Modulation," MILCOM, Boston, 1993, pp. $447-450$.

[3] A. Batra et al., "Multi-band OFDM Physical Layer Proposal for IEEE 802.15 Task Group 3a," IEEE P802.15-03/142r2-TG3a, Sep. 2004.

[4] Available: http://ieee802.org/15/pub/TG3.html.

[5] E. Saberinia and A. H. Tewfik, "Multi-user UWB-OFDM communications," Proc. 2003 IEEE Pacific Rim Conf. on Communications, Computersand Signal Processing, vol. 1, pp. 127-130, Aug. 2003.

[6] A. Batra, J. Balakrishnan, A. Dabak, et al., "TI Physical Layer Proposal for IEEE 802.15 Task Group 3a," IEEE P802.15-03/142r2-TG30, March 2003.

[7] M Abramovitz and I.A. Stegun, "Orthogonal polynomials, " in Handbook of mathematical functions with formulas, graphs and mathematical tables, ed. Dover, New York, 1972. 
[8] F. Elbahhar, A. Rivenq-Menha j, J.M. Rouvaen, M. Heddebaut and T. Boukour, Comparison between DS-CDMA and Modified Gegenbauer Functions for a multi-user communication Ultra Wide Band system, I.E.E. Proceedings Communications, vol. 152, pp. 1021-1028, 2005.

[9] F.Elbahhar. " Multi-user Ultra Wide Band communication system based on modified Gegenbauer and Hermite functions," PhD. University of Valenciennes, May 2004.

[10] F. Elbahhar, A. Rivenq-Menhaj et J.M Rouvaen, Multi-user Ultra Wide Band communication system based on modified Gegenbauer and Hermite functions, Wireless Personal Communications (Kluwer), vol. 34, pp. 255$277,2005$.

[11] D. C. Popescu and P. Yaddanapudi, Narrowband Interference Avoidance in OFDM-Based UWB Communication Systems, IEEE Transactions on Communications, accepted March 2006, in press.

[12] W. C. Lindsey and M. K. Simon, "Telecommunication Systems Engineering," Prentice-Hall, Englewood Cliffs, NJ, 1973.

[13] V. K. Prabhu, "PSK performance with imperfect carrier recovery," IEEE Trans. Aerosp. Electron. Syst., vol. 12, no. 2, pp. 275-285, Mar. 1976.

[14] A. J. Viterbi, "Principles of Coherent Communication." McGraw-Hill, New York, 1966.

[15] J.G. Proakis, Digital communications, ed. Mc-Graw Hill, New York, 2001.

[16] T. S. Rappaport, "Wireless Communications Principles and Practice," 2nd Edition, Prentice Hall Inc, 2002.

[17] J. K. Tugnait, L. Tong, Z. Ding, "Single-user channel estimation and equalization," IEEE Signal Processing Mag., 17, 16-28, 2000.

[18] J. G. Proakis, C. L. Nikias, "Blind equalization (overview paper)," Proceedings of SPIE, 28, 76-87, 1991.

[19] S. Verdu, Minimum Probability of Error for Asynchronous Gaussian Multiple-Access Channels, IEEE Trans, info. Theory, vol. IT-32, no. 1, pages 85-96, Janvier 1986.

[20] M.K. Varanasi , B. Aazhang, "Multistage detection in asynchronous codedivision multiple-access communications," IEEE Trans. Commun., COM38, 509-519, 1990.

[21] D. Divsalar, M. Simon, D. Raphaeli, "Improved parallel interference cancellation for CDMA," IEEE Trans. Commun., 46, 258-268, 1998.

[22] J. M. Holtzman, "CDMA Power Control for Wireless Networks Third Generation," in Wireless Information Networks, Kluwer, 1992. 
[23] R. E. Ziemer, Roger L. Peterson, "Introduction to Digital Communication," Macmillan, New York, 1992.

[24] R.A. Cameron, B.D. Woerner, "Performance analysis of CDMA with imperfect power control," IEEE Trans. Commun., vol. 44, 777-781, 1996. 